\title{
Anti-invasion and anti-migration effects of miR-199a-3p in hepatocellular carcinoma are due in part to targeting CD151
}

\author{
JI HYE KIM ${ }^{1 *}$, MOHAMED BADAWI ${ }^{1 *}$, JONG-KOOK PARK ${ }^{1,5}$, JINMAI JIANG ${ }^{1,6}$, \\ XIAOKUI MO ${ }^{2}$, LEWIS R. ROBERTS ${ }^{3}$ and THOMAS D. SCHMITTGEN ${ }^{1,4}$ \\ ${ }^{1}$ College of Pharmacy, ${ }^{2}$ Department of Biostatistics, Ohio State University, Columbus, OH; \\ ${ }^{3}$ Divisions of Gastroenterology and Hepatology, Mayo Clinic College of Medicine, Rochester, MN, USA
}

Received March 30, 2016; Accepted June 13, 2016

DOI: 10.3892/ijo.2016.3677

\begin{abstract}
Several studies have reported reduced miR-199a-3p expression in hepatocellular carcinoma (HCC). In an effort to discover important target genes for miR-199a-3p that may be related to $\mathrm{HCC}$ development or progression, we identified the tetraspanin, transmembrane glycoprotein CD151. Luciferase reporter assays and western blotting identified CD151 as a bona fide miR-199a-3p target gene. While CD151 protein was increased in the mesenchymal but not the epithelial HCC cell lines, CD151 knockdown with siRNA did not reduce HCC cell proliferation in either group of cells. miR-199a-3p reduced in vitro invasion and migration of CD151-positive HCC cells. Examination of the mRNA and protein expression in pairs of primary HCC tumors and adjacent benign tissues showed that not only was CD151 mRNA and protein increased in the tumors but also that an inverse correlation exists between the miR-199a-3p and CD151 RNA expression. We report that CD151 is a target of miR-199a-3p and that increased CD151 protein resulting from reduced miR-199a-3p could contribute to the development of metastatic HCC.
\end{abstract}

\section{Introduction}

Hepatocellular carcinoma (HCC) is the second most common cause of cancer death worldwide, accounting for an estimated 745,000 deaths per year, representing $10 \%$ of all deaths from cancer (1). Major risk factors for HCC include hepatitis C

Correspondence to: Dr Thomas D. Schmittgen, ${ }^{4}$ Present address: College of Pharmacy, University of Florida, Gainesville, FL 32610, USA

E-mail: tschmittgen@ufl.edu

Present addresses: ${ }^{5}$ Department of Dermatology, Northwestern University, Chicago, IL, USA; ${ }^{6}$ College of Pharmacy, University of Florida, Gainesville, FL, USA

\section{${ }^{*}$ Contributed equally}

Key words: liver, microRNA, Tspan24, metastasis, miR-199a* virus (HCV) or hepatitis B virus (HBV) infection, alcoholic cirrhosis and nonalcoholic fatty liver disease. Resection, local ablation or transplantation are effective treatments for early stage HCC. Transarterial chemoembolization shows limited success for intermediate stage HCC without invasion or metastasis (2). However, the overall survival for advanced $\mathrm{HCC}$ is poor due to rapid tumor progression and metastasis (3). Therefore, it is necessary to better understand the mechanisms of HCC metastasis and to develop new therapeutic options for advanced or recurrent $\mathrm{HCC}$.

It has been shown that expression of miRNAs is dysregulated in all cancers (4). miRNAs may play an oncogenic or tumor suppressive role depending on the type of cancer. miR-199a-3p is a miRNA that displays decreased expression in HCC (5-8). The genes that encode miR-199a-1 and miR-199a-2 are located within introns of the DNM2 and DNM3 genes, respectively. Previous research has shown that miR-199a-3p regulates expression of c-MET $(9,10)$, mTOR (9) and PAK4 (6). We previously reported that CD44 is a target of miR-199a-3p in HCC (11).

CD151 (Tspan24) is a member of the tetraspanin protein family that have been linked to metastasis (12-14). CD151 is associated with proMMP7 and proMMP9 transcription which facilitates matrix degradation and regulates cell migration. Several studies have demonstrated that CD151 is involved in the regulation of pathways downstream of the hepatocyte growth factor (HGF)/c-Met axis (15) and CD151 was remarkably overexpressed in HCC (12). High expression levels of CD151 and integrin subunit $\alpha 6$ increased invasiveness of HCC cells (14) and overexpression of CD151 promoted the expression of MMP9, which is one of the key factors in metastasis through the PI3K/Akt/GSK-3 $\beta /$ Snail pathway (13). Recent studies showed that CD151 expression could be regulated by miRNAs. miR-506 suppressed CD151 in a breast cancer cell line (16) and miR-124 inhibits invasiveness and metastatic potential of breast cancer cells by targeting CD151 mRNA (17). In addition, miR-22 reduces cell proliferation and invasiveness of gastric cancers by suppressing CD151 (18).

We confirm previous findings that low miR-199a-1 expression is correlated with poor survival in HCC and that miR-199a-3p is significantly downregulated in HCC (5-8). We report that CD151 is a direct target of miR-199a-3p and 
that reintroduction of miR-199a-3p to HCC cells strikingly suppressed cell migration and invasion in vitro in part by targeting CD151.

\section{Materials and methods}

Cell line and tissue specimens. The human HCC cell lines SNU-423, SNU-449, PLC/PRF/5, HepG2, Hep3B and SK-Hep-1, were purchased from American Type Tissue Collection (Manassas, VA, USA). SNU-423, SNU-449 cells were cultured in RPMI-1640 medium (Invitrogen) with $10 \%$ fetal bovine serum (Sigma). PLC/PRF/5, Hep3B, HepG2 and SK-Hep-1 were cultured in MEM medium (Invitrogen) with $10 \%$ fetal bovine serum (Sigma). All cell lines were successfully authenticated by the Interdisciplinary Center for Biotechnology Research at the University of Florida (data not shown). Eighteen paired HCC and adjacent non-tumor liver tissues were collected from patients during surgical resections at the Mayo Clinic (Rochester, MN, USA), frozen in liquid nitrogen and stored at $-80^{\circ}$ until RNA and protein were extracted. Sample collection conformed to the policies and practices of the facility's Institutional Review Board. Patient demographics are presented in Table I. The TNM classification for hepatocellular carcinoma was used for tumor stage and grade.

Transfection of microRNA mimic and siRNA oligonucleotides. HCC cell lines were transfected either with $100 \mathrm{nM}$ of hsa-miR-199a-3p mimic or negative control (Ambion), or with $100 \mathrm{nM}$ of CD151 siRNA or control siRNA (Thermo Scientific) using Lipofectamine 2000 (Invitrogen) and Opti-MEM medium (Invitrogen). Cells were transfected with the miRNA mimic or siRNA oligonucleotides for $72 \mathrm{~h}$ prior to extraction of RNA or protein.

RNA extraction, $c D N A$ synthesis and $q R T-P C R$. Total RNA was extracted from 18 pairs of HCC tumors and adjacent benign liver tissue specimens. Following pulverization in a cold mortar and pestle, total RNA was isolated from the tissues using TRIzol reagent (Life Technologies). cDNA was synthesized as previously described (19). Five hundred nanograms of total RNA was used to synthesize cDNA using random primers. cDNA was analyzed for gene expression using gene specific primers (IDT) and the Express SYBR ${ }^{\circledR}$ GreenER qPCR super mix (Invitrogen). For the miRNA expression analysis, cDNA primed with $100 \mathrm{ng}$ of total RNA was assayed using the TaqMan ${ }^{\circledR}$ microRNA assays (Applied Biosystems) as described (20). Data were normalized to $18 \mathrm{~S}$ rRNA and the relative expression of genes was presented using the comparative $\mathrm{C}_{\mathrm{T}}$ method. Data were multiplied by $10^{6}$ to simplify presentation. Primer sequences are available upon request.

Dual-luciferase reporter gene assay. The full length CD151 3'UTR was cloned into the psiCHECK-2 Vector (Promega). Three nucleotides in the binding sequences of CD151 3'UTR was mutated by QuikChange XL Site-Directed Mutagenesis kit (Agilent Technologies) and the mutation was confirmed by sequencing. The luciferase reporter gene assay was performed using the Dual-luciferase reporter assay system (Promega) according to the manufacturer's instructions. SNU-449 cells were plated at a density of 50,000 cells/well (24-well). Following a 24-h incubation, cells were co-transfected with miR-199a-3p mimic or negative control oligonucleotide $(50 \mathrm{nM})$ along with the WT-CD151 3'UTR or mutated CD151 3'UTR constructs using Lipofectamine 2000 (Invitrogen). Reporter gene assays were performed $48 \mathrm{~h}$ post-transfection using the Dual luciferase assay system (Promega). Renilla luciferase activity was normalized for transfection efficiency using the corresponding Firefly luciferase activity. All experiments were performed at least three times.

Cell proliferation assay. PLC/PRF/5, SK-Hep-1, SNU-423 and SNU-449 cells were seeded at a density of 2,000 cells per well in 96-well culture plates. Following a 24-h incubation period, cells were transfected with $100 \mathrm{nM}$ of CD151 siRNA or control siRNA oligonucleotides. Cell proliferation was determined $96 \mathrm{~h}$ later using the WST-1 reagent (Roche) per the manufacturer's recommendations. All experiments were performed at least in triplicate.

Cell migration assay. A wound healing assay was performed to evaluate cell migration in vitro. SNU-449 cells were transfected with either $100 \mathrm{nM}$ of miR-199a-3p mimic or negative control oligonucleotides, or $100 \mathrm{nM}$ of CD151 siRNA or control siRNA. Twenty-four hours after transfection, $70 \mu \mathrm{l}$ of the transfected cells $\left(3 \times 10^{5}\right.$ cells $\left./ \mathrm{ml}\right)$ was placed into each well of an ibidi culture-insert (ibidi, LLC). After an overnight incubation, the culture insert was removed to create a cell-free gap in the monolayer. The gap closure area was photographed and analyzed by TScratch software (21). The percentage of the gap area closed between the beginning and end of the experiment was calculated from at least three independent experiments.

Matrigel invasion assay. In vitro cell invasion assays were conducted using the CytoSelect ${ }^{\mathrm{TM}}$ 24-well cell invasion assay kit ( $8-\mu \mathrm{m}$ pore size, Cell Biolabs, Inc.). SNU-449 cells were transfected with either $100 \mathrm{nM}$ of miR-199a-3p mimic or negative control oligonucleotides, or $100 \mathrm{nM}$ of CD151 siRNA or control siRNA. Forty-eight hours after transfection, the transfected cells were placed into the upper chamber at a density of $1.5 \times 10^{5}$ cells per well in $1 \%$ FBS containing medium. Ten percent FBS containing medium was placed in the lower chamber as a chemoattractant. Cells were incubated at $37^{\circ} \mathrm{C}$ for $24 \mathrm{~h}$ and the cells that invaded the membrane were fixed and stained. The number of cells invading the membrane were counted in three different fields per experiment.

Protein extraction and immunoblotting. Cell protein lysates in RIPA buffer (Sigma) were separated on NuPAGE 4-12\% Bis-Tris gels (Novex) and electrophoretically transferred to polyvinylidene difluoride membranes (Roche). The blotting was performed for CD151 (ab33315, Abcam). $\beta$-actin (Abcam) or GAPDH (sc-32233, Santa Cruz Biotechnology) were used as loading controls. Secondary horseradish peroxidase antibody was detected using the ECL Western Blotting Analysis system (Amersham Biosciences).

Statistical analysis. The matched samples were compared using paired t-tests and samples subjected to different treat- 
Table I. Patient data.

\begin{tabular}{|c|c|c|c|c|c|c|c|c|}
\hline Patient & Gender & Age & Grade & Stage & $\begin{array}{l}\text { Tumor size }(\mathrm{cm}) \\
\text { (largest mass) }\end{array}$ & Cirrhosis & $\begin{array}{l}\text { Fibrosis } \\
\text { stage }\end{array}$ & Etiology \\
\hline 10 & $\mathrm{~F}$ & 62.5 & 2 & IV & 22 & No & 0 & No known risk factors \\
\hline 14 & M & 89.1 & 2 & IV & 6.5 & No & 0 & Hereditary hemochromatosis \\
\hline 26 & M & 43.3 & 1 & I & & No & 0 & No known risk factors \\
\hline 33 & $\mathrm{~F}$ & 43.2 & 2 & I & & Yes & 4 & $\mathrm{HCV}$ \\
\hline 44 & $\mathrm{~F}$ & 48.0 & 1 & I & & No & 0 & No known risk factors \\
\hline 69 & $\mathrm{~F}$ & 85.0 & 1 & $\mathrm{I}$ & & No & 0 & No known risk factors \\
\hline 92 & M & 76.2 & 2 & II & & Yes & 4 & Autoimmune hepatitis \\
\hline 94 & $\mathrm{~F}$ & 82.7 & 3 & II & & No & 0 & No known risk factors \\
\hline 97 & $\mathrm{~F}$ & 50.8 & 2 & I & 10.5 & No & 0 & No known risk factors \\
\hline 99 & $\mathrm{~F}$ & 83.3 & 3 & $\mathrm{I}$ & & Yes & 4 & $\mathrm{HCV}$ \\
\hline 103 & $\mathrm{~F}$ & 84.7 & 2 & II & 8 & No & 0 & Alcohol \\
\hline 106 & M & 59.9 & 2 & $\mathrm{I}$ & & No & 0 & Alcohol \\
\hline 109 & M & 80.3 & 2 & $\mathrm{I}$ & 6 & No & 0 & No known risk factors \\
\hline 306 & M & 72.3 & 2 & II & 4.3 & Yes & 4 & No known risk factors \\
\hline 309 & M & 81.2 & 3 & I & 5 & No & 0 & $\mathrm{HCV}$ probable remote history $\mathrm{HCB}$ \\
\hline 337 & M & 80.7 & 3 & II & 14.4 & Yes & 4 & Alcohol \\
\hline 361 & M & 71.0 & 3 & I & 10.8 & No & 0 & No known risk factors \\
\hline 367 & M & 71.5 & 2 & II & 4 & No & 2 & $\mathrm{HCB}$ \\
\hline
\end{tabular}

ments were compared using a Student's 2-sample t-tests. A $\mathrm{p}<0.05$ was considered significant. The Cancer Genome Atlas (TCGA) microRNA-seq expression data and patients' clinical information $(n=141)$ were downloaded through the TCGA data portal. Patients were dichotomized into two groups (high and low) according to the median expression of miR-199-1 or miR-199-2. The probabilities of 5-year survival between groups were compared by using the Kaplan-Meier method and log-rank test. Data analysis was performed using SAS 9.4 (SAS, Inc; Cary, NC, USA). For the analysis of miR-199-1 expression in paired benign and HCC, data from 49 pairs of benign and HCC were used. This represents all of the paired specimen data for HCC on the TCGA data portal.

\section{Results}

CD151 is a target of miR-199a-3p in HCC. To better understand the role of miR-199a-3p in HCC, we searched potential miR-199a-3p targets using the TargetScan algorithm. A putative miR-199a-3p binding site within the CD151 3'UTR was highly conserved (Fig. 1A). A luciferase reporter assay was used to confirm the binding of miR-199a-3p to the CD151 3'UTR. Luciferase expression was reduced by $>50 \%$ by miR-199a-3p mimic (Fig. 1B). The luciferase expression was not reduced substantially when the miR-199a-3p binding site on the CD151 3'UTR was mutated (Fig. 1B). To further investigate if miR-199a-3p functionally regulates CD151, SNU-423 and SNU-449 cells were transfected with miR-199a-3p mimic or negative control oligonucleotides. Western blotting experiments showed that the protein expression of CD151 was reduced by $60 \%$ in SNU-423 and by $43 \%$ in SNU-449 cells compared to control (Fig. 1C). qRT-PCR showed that miR-199a-3p mimic reduced CD151 mRNA in these cell lines (Fig. 1D). Together these data indicate that CD151 is a direct target of miR-199a-3p and that the miR-199a-3p binding enhances CD151 mRNA degradation.

Attenuation of CD151 does not reduce HCC proliferation. CD151 protein as well as several epithelial-mesenchymal transition (EMT) markers were examined in $\mathrm{HCC}$ cell lines. HepG2, Hep3B and PLC/PRF/5 express high levels of CDH1, an epithelial marker whereas SNU-449, SNU-423 and SK-Hep-1 express high levels of the mesenchymal markers VIM, ZEB2 and TWIST (Fig. 2A-D). Interestingly, CD151 protein was overexpressed in the mesenchymal-like cell lines (SNU-449, SNU-423 and SK-Hep-1) compared to cell lines expressing epithelial genes (HepG2, Hep3B and PLC/PRF/5) (Fig. 2E), suggesting CD151 is associated with the mesenchymal phenotype. To determine if a direct relationship exists between mesenchymal markers and CD151, the expression of epithelial (CDH1) and mesenchymal (VIM, CDH2 and ZEB1) was measured in SNU-423 cells following siRNA knockdown of CD151. Knockdown of CD151 by $\leq 80 \%$ did not significantly alter the expression of CDH1, VIM, CDH2 or ZEB1 (data not shown).

To determine if CD151 regulates cell proliferation, we transfected four different HCC cell lines with CD151 or control siRNA. Knockdown of CD151 by $>80 \%$ failed to reduce cell proliferation in either CD151-negative (PLC/PRF/5) or CD151positive (SK-Hep-1, SNU-423 and SNU-449) cells (Fig. 2F). These results suggest that $\mathrm{CD} 151$ is not involved in regulating HCC cellular proliferation. 
A

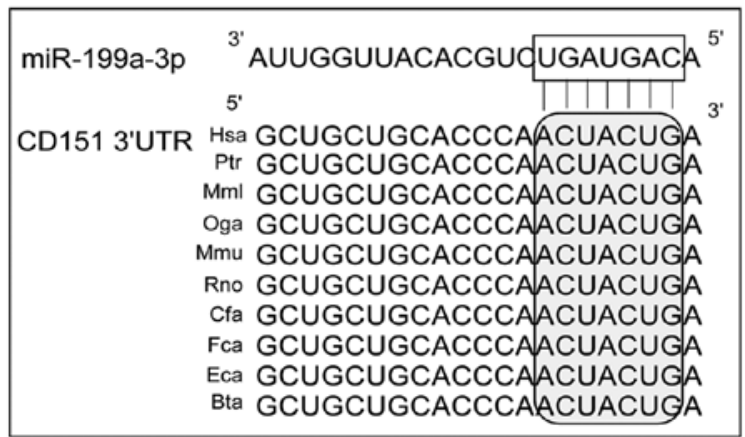

C

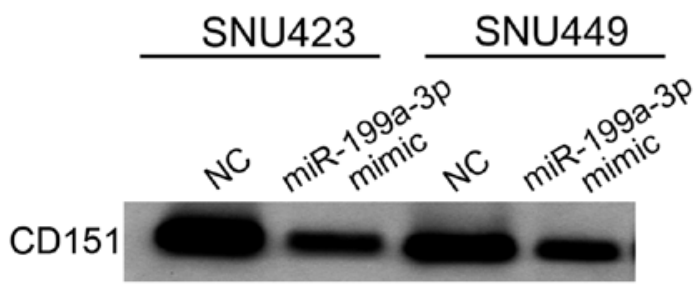

$\beta$-actin
B

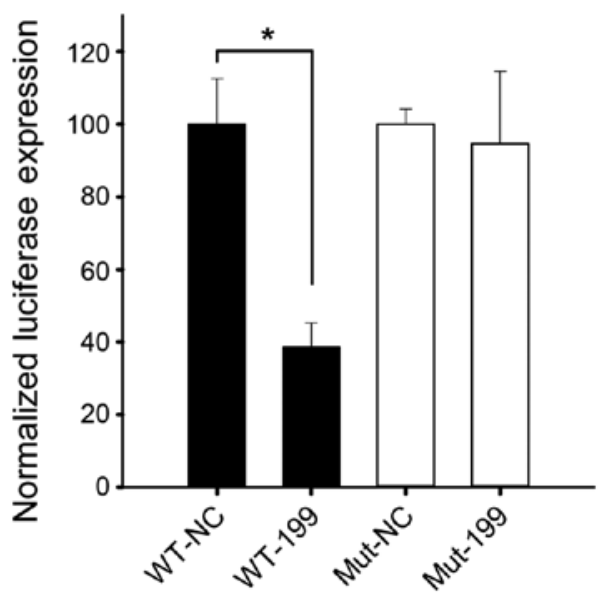

D

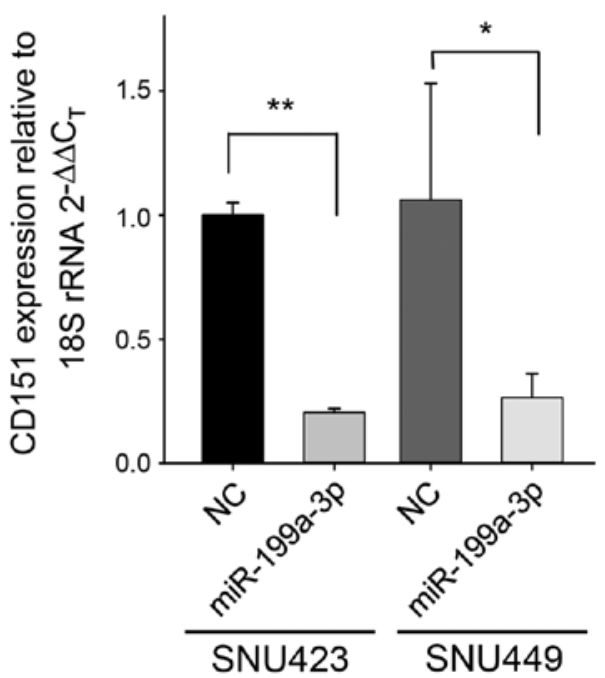

Figure 1. CD151 is a target of miR-199a-3p. (A) Schematic diagram representing the location and conservation of the putative miR-199a-3p binding site within the 3'UTR of CD151 mRNA. (B) Luciferase reporter plasmids containing the wild-type or mutated CD151 3'UTR were transiently transfected into the SNU-449 cell line with $50 \mathrm{nM}$ of miRNA-199a-3p mimic control or miR-199a-3p oligonucleotides. Luciferase expression was measured $48 \mathrm{~h}$ after transfection. Normalized Renilla luciferase activity in cells transfected with negative control oligonucleotides (NC) was set at $100 \%$. The data represent the mean \pm SD of at least 3 independent transfections. (C) Immunoblot of CD151 protein expression in SNU-423 and SNU-449 cells transfected with control oligonucleotide or miR-199a-3p mimic, (D) Relative CD151 mRNA expression was measured by qRT-PCR in control oligonucleotide or miR-199a-3p mimic transfected cells.

In vitro cell migration and invasion is inhibited by miR-199a-3p through targeting CD151. Next we determined whether miR-199a-3p mimic could inhibit in vitro cell migration and invasion under conditions of CD151 suppression. CD151positive SNU-449 and SNU-423 cells were transfected with miR-199a-3p mimic under the identical conditions shown to suppress CD151 mRNA and protein expression. As a control, wound healing and invasion assays were performed following transfection with CD151 or control siRNA. Compared to control oligonucleotide, wound healing was decreased in SNU-449 cells after transfection of miR-199a-3p mimic (Fig. 3A and B). Wound healing was also significantly reduced after CD151 siRNA transfection compared to control siRNA (Fig. 3C and D). We also repeated the wound healing experiment in a second cell line (SNU-423 cells) with similar results (Fig. 3E). In addition, the number of invading SNU-449 cells was strikingly reduced after transfection with miR-199a-3p mimic (Fig. 4A and B). Cell invasiveness was also significantly suppressed after CD151 siRNA transfection (Fig. 4C and D).
The results were reproduced in SNU-423 cells (Fig. 4E). These data suggest that suppression of CD151 expression by miR199a-3p mimic can reduce cell migration and invasion in vitro.

miR-199a-3p and CD151 expression inversely correlates in HCC specimens. The expression of miR-199a-3p was measured in 18 pairs of human HCC tissues and adjacent benign tissues by qRT-PCR. miR-199a-3p was significantly downregulated in the HCC tissues compared to the adjacent benign liver $(\mathrm{p}<0.0001$, paired t-test, Fig. $5 \mathrm{~A})$, confirming previous results $(5-8)$. We also evaluated the expression of miR-199a-1 and miR-199a-2 in 49 pairs of tumor and adjacent benign from the TCGA data set. miR-199a-1 and miR-199a-2 are two isogenic genes encoding miR-199a-3p. The expression of miR-199a-1 (Fig. 5B, p<0.0001) and miR-199a-2 (p<0.0001, not shown) was reduced in the tumor compared to the adjacent benign tissue. Next, we investigated the correlation between miR-199a gene expression and survival by analyzing data from the TCGA database. HCC patients with high miR-199a-1 
A

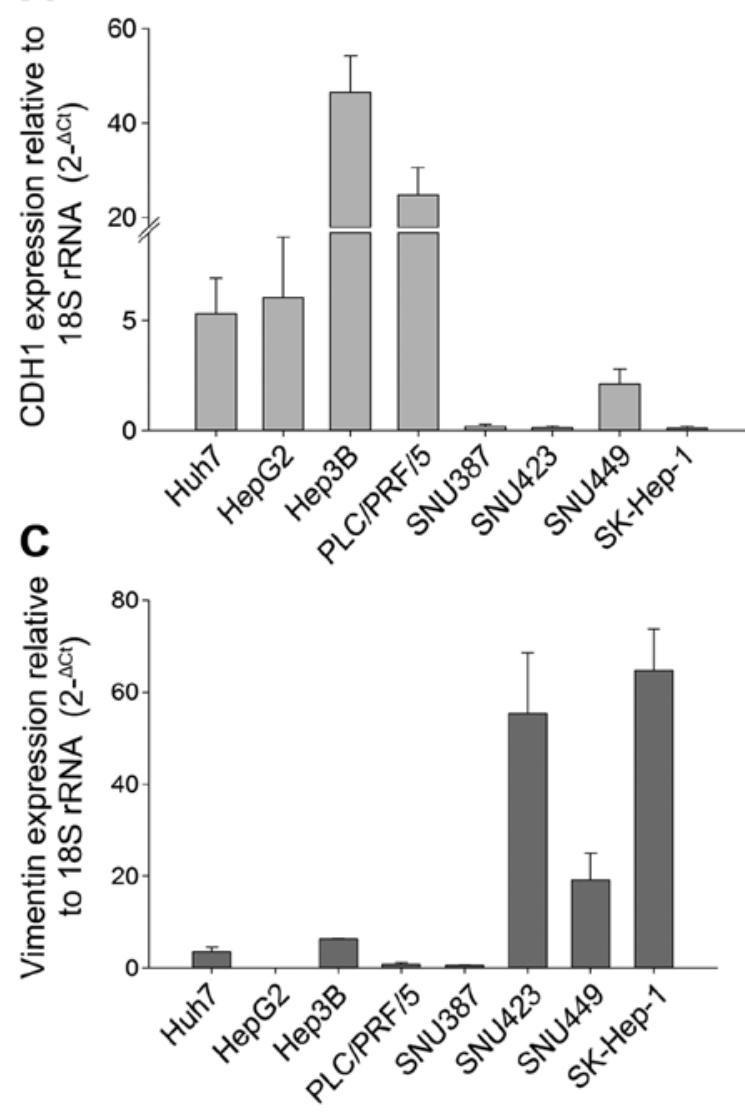

E

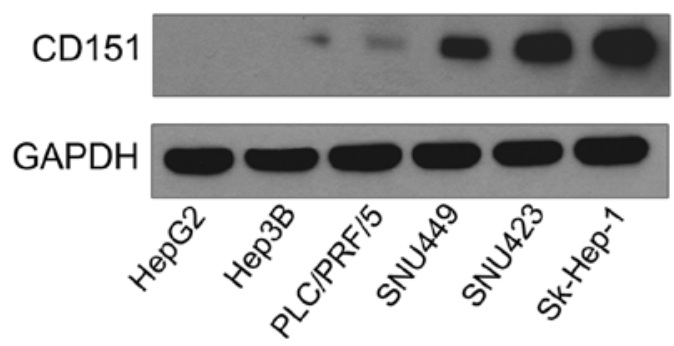

B
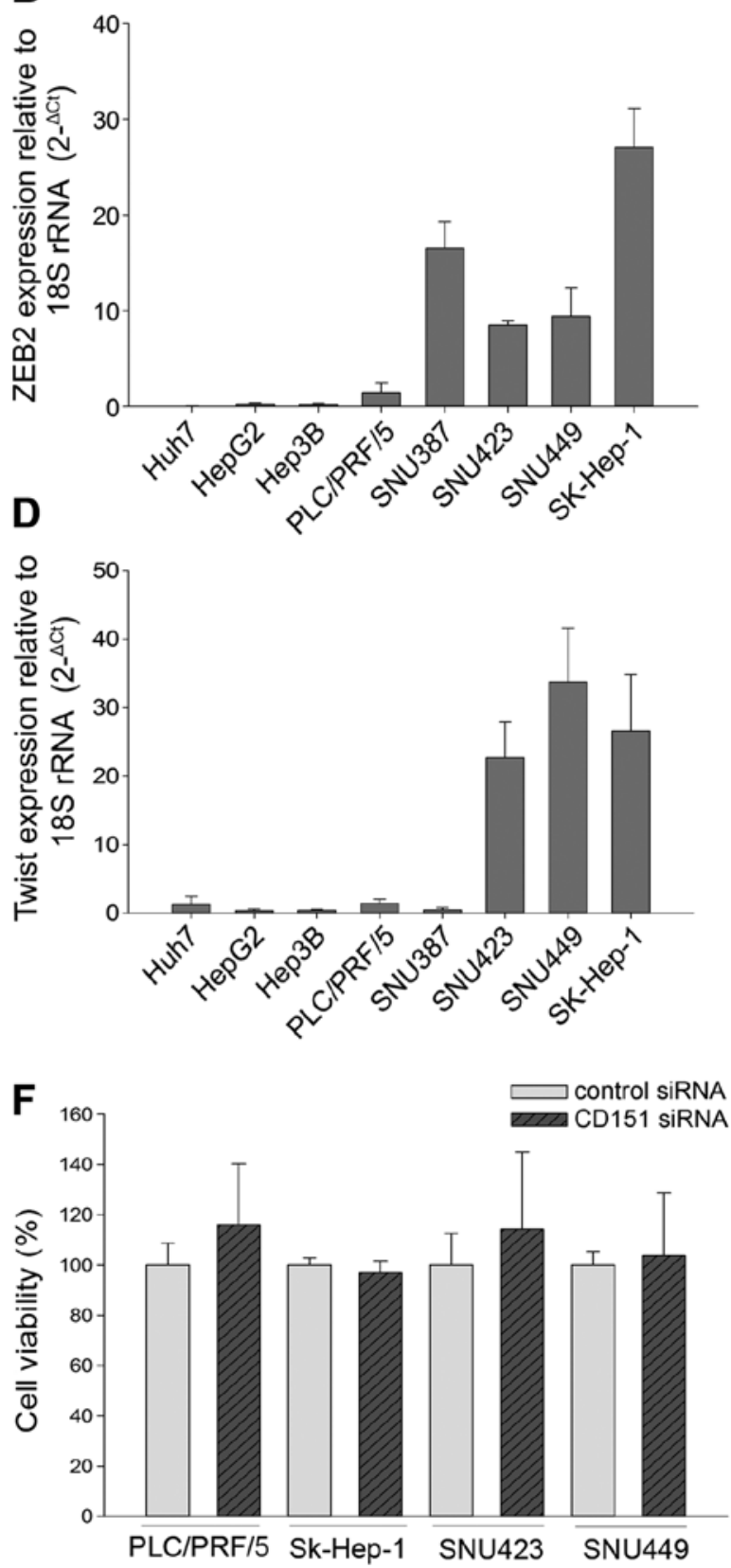

Figure 2. CD151 is overexpressed in mesenchymal HCC cell lines and is not involved in cell proliferation in vitro. Several epithelial [(A) CDH1] and mesenchymal [(B) ZEB1, (C) VIM and (D) TWIST] markers were analyzed in HCC cell lines by qRT-PCR. (E) CD151 protein levels were assayed by western blotting in HCC cell lines. (F) HCC cell lines were transfected with $100 \mathrm{nM}$ control siRNA or CD151 siRNA. Proliferation was determined by WST-1 assay $96 \mathrm{~h}$ after transfection.

expression had better survival compared to those with low miR-199a-1 levels ( $<<0.05$, Fig. 5C). While there was good separation between survival and miR-199a-2 expression, the correlation was not significant ( $\mathrm{p}=0.3$, data not shown).

CD151 mRNA (qRT-PCR) and protein (western blotting) were also examined in paired specimens of $\mathrm{HCC}$ and adjacent benign liver. CD151 mRNA was upregulated in HCC tissues compared to paired benign tissues $(\mathrm{p}<0.001$, paired t-test, Fig. 6A). Expression of CD151 was also increased in the paired HCC tissues from the TCGA data (data not shown). Moreover, CD151 protein expression was strongly overexpressed in HCC tissues (Fig. 6B). Finally, using Pearson correlation analysis, we found a strong inverse correlation between CD151 mRNA and miR-199a-3p expression in HCC (Fig. 6C).

\section{Discussion}

Several studies have reported reduced expression of miR-199a in $\mathrm{HCC}(6-8,22,23)$. We confirm these results in a new cohort of HCC patients (Fig. 5A) and in the TCGA data set (Fig. 5B). We show that low expression of miR-199a-1 (Fig. 5C) correlates with poor survival in HCC, confirming the data of Fornari et al (9). Furthermore, we report that miR-199a-3p regulates CD151. CD151 is a tetraspanin protein family member and has been 

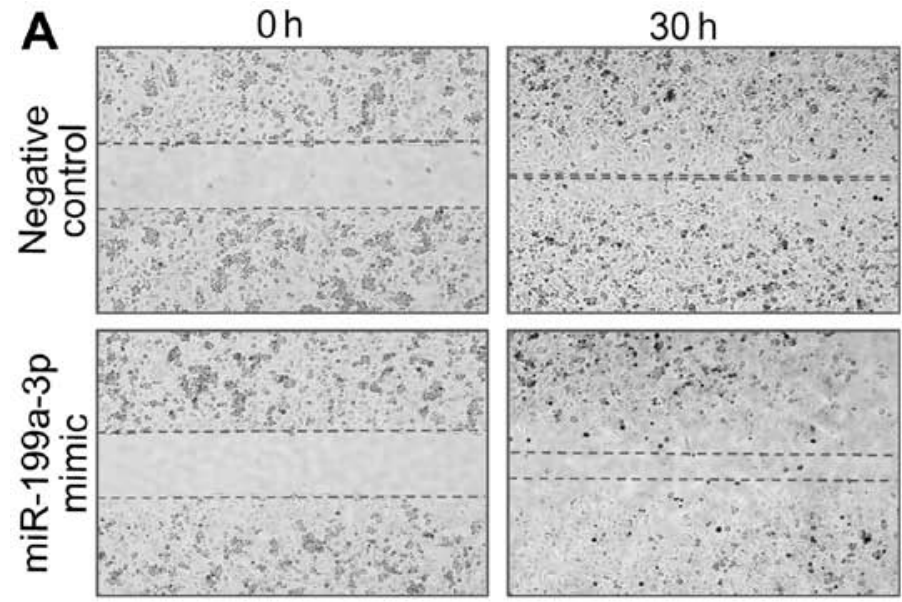

B
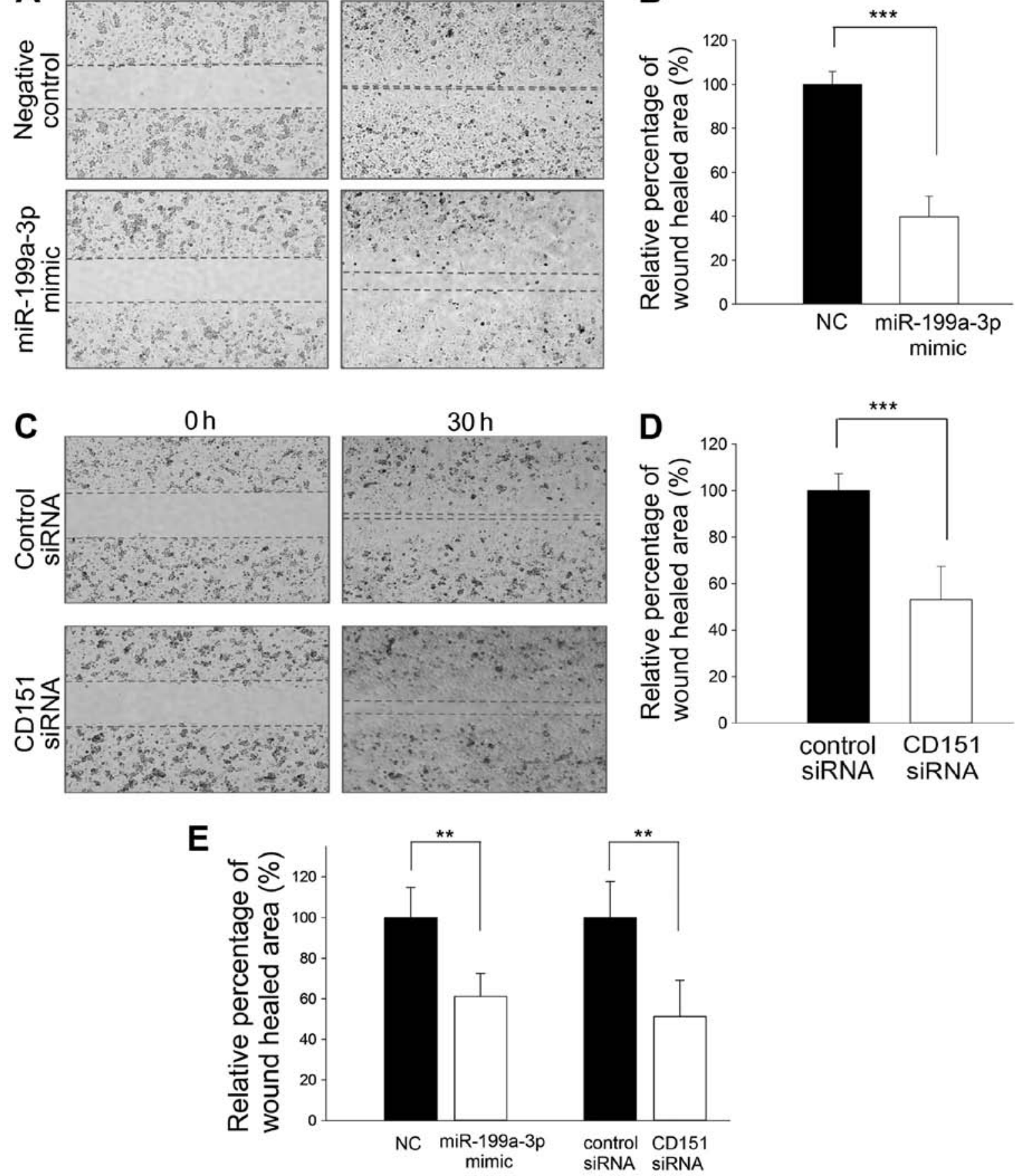

Figure 3. Reintroduction of miR-199a-3p and knockdown of CD151 reduces cell migration in vitro. (A and B) Wound healing assays were conducted with negative control and miR-199a-3p mimic transfected SNU-449 cells (4x magnification). (C and D) Wound healing assays were conducted with control siRNA or CD151 siRNA transfected SNU-449 cells (4x magnification). (E) Wound healing assay in SNU-423 cells transfected with miR-199a-3p mimic, siRNA to $\mathrm{CD} 151$ or control oligonucleotides. The data are representative of three independent experiments; ${ }^{* *} \mathrm{p}<0.01,{ }^{* * *} \mathrm{p}<0.001$.

implicated in HCC invasion and migration (12-14). The role of CD151 in HCC invasion and metastasis is believed to rely on its ability to form complexes with laminin-binding integrin receptors $(\alpha 6 \beta 1, \alpha 6 \beta 4$ and $\alpha 3 \beta 1)(14,24)$ as well as regulate cell-cell and cell-matrix interactions (25). CD151 was previously associated with the mesenchymal phenotype in HCC (14). Knockdown of CD151 in CD151-positive HCCLM3 cells, or overexpression of CD151 in CD151-negative HepG2 cells, altered the mesenchymal phenotype when these cells were cultured along with laminin 5 (14). Induction of EMT occurred through hyperactivation of the PI3K-Akt-Snail and PTEN feedback pathway (14). Successful knockdown of CD151 did not alter the expression of EMT markers, however our cells were not cultured along with laminin 5 as previously shown (14).

We report that CD151 mRNA and protein are increased in HCC patients. Liver cirrhosis is one predisposing factor to HCC. We compared the expression of miR-199a-3p and CD151 in cirrhotic and non-cirrhotic HCC patients to determine if a relationship exists. Thirty-six percent (\%) of the patients in our 

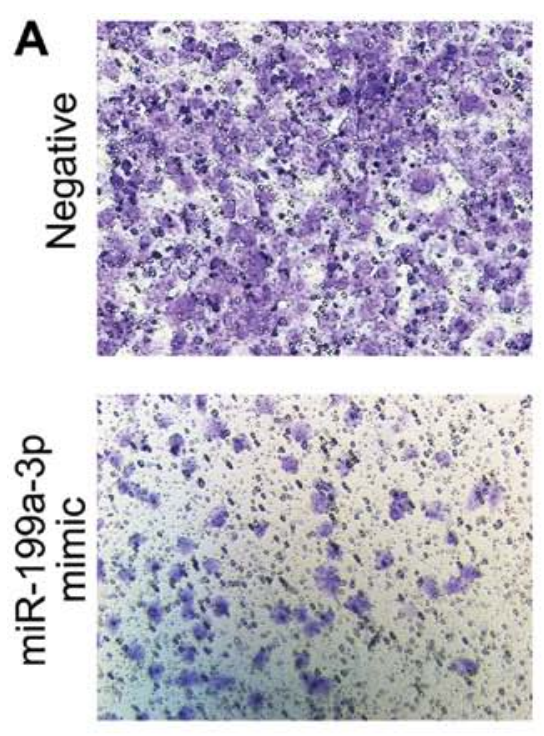

B
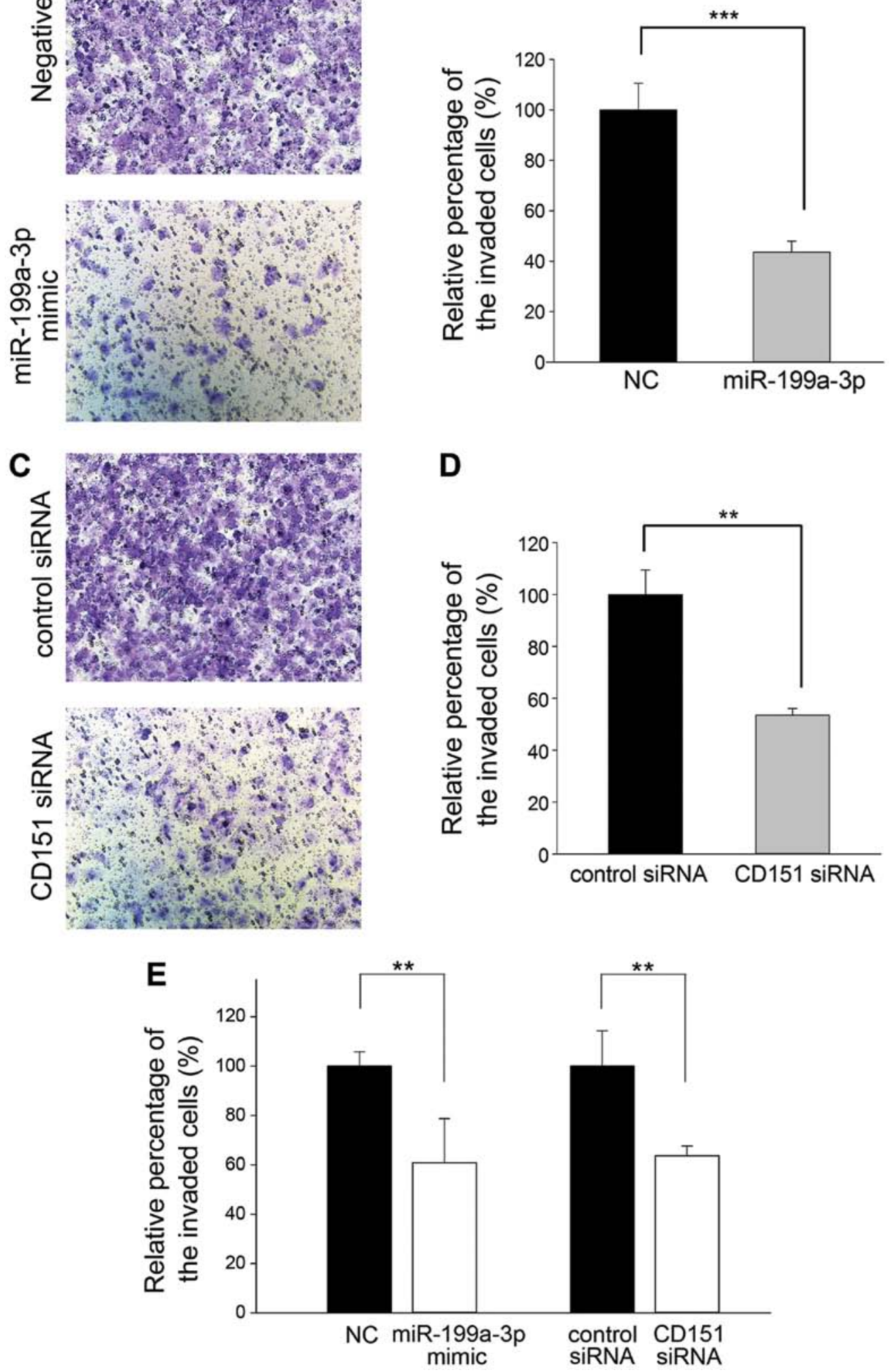

Figure 4. Reintroduction of miR-199a-3p and knockdown of CD151 reduces cell invasion in vitro. (A and B) Boyden chamber invasion assays were conducted with negative control and miR-199a-3p mimic transfected SNU-449 cells. (C and D) Boyden chamber invasion assays were conducted with control siRNA or CD151 siRNA transfected SNU-449 cells. (E) Invasion assay in SNU-423 cells transfected with miR-199a-3p mimic, siRNA to CD151 or control oligonucleotides. The number of the invading cells was determined from 5 different fields for each experiment; ${ }^{* *} \mathrm{p}<0.01,{ }^{* * * *} \mathrm{p}<0.001$.

data set had cirrhosis (Table I), however there was no correlation between CD151 or miR-199a-3p and cirrhosis (data not shown). Also no correlation existed between miR-199a-3p and
CD151 in the TCGA data set (data not shown). The regulation of CD151 by miR-199a-3p was further confirmed in HCC patients by the strong negative correlation between these two 


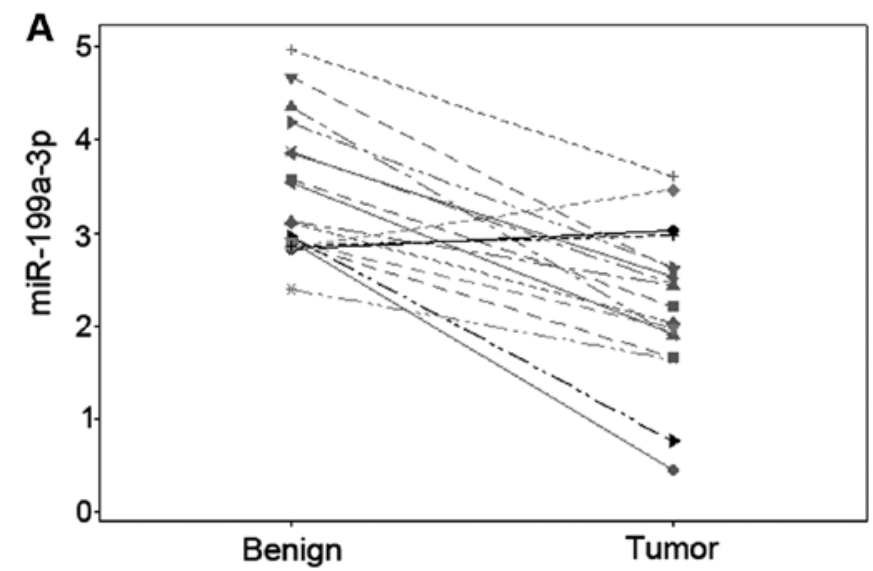

A
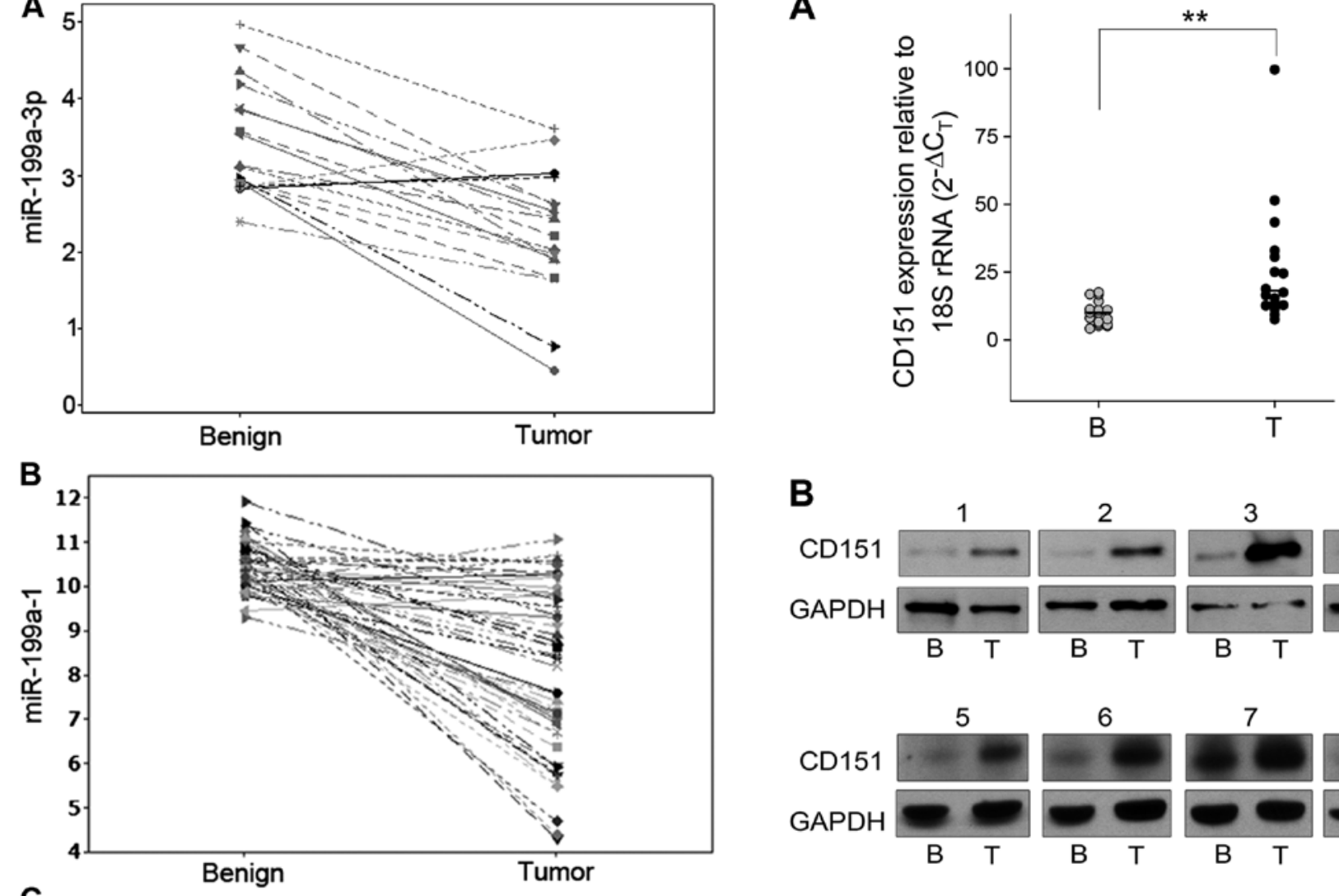

B
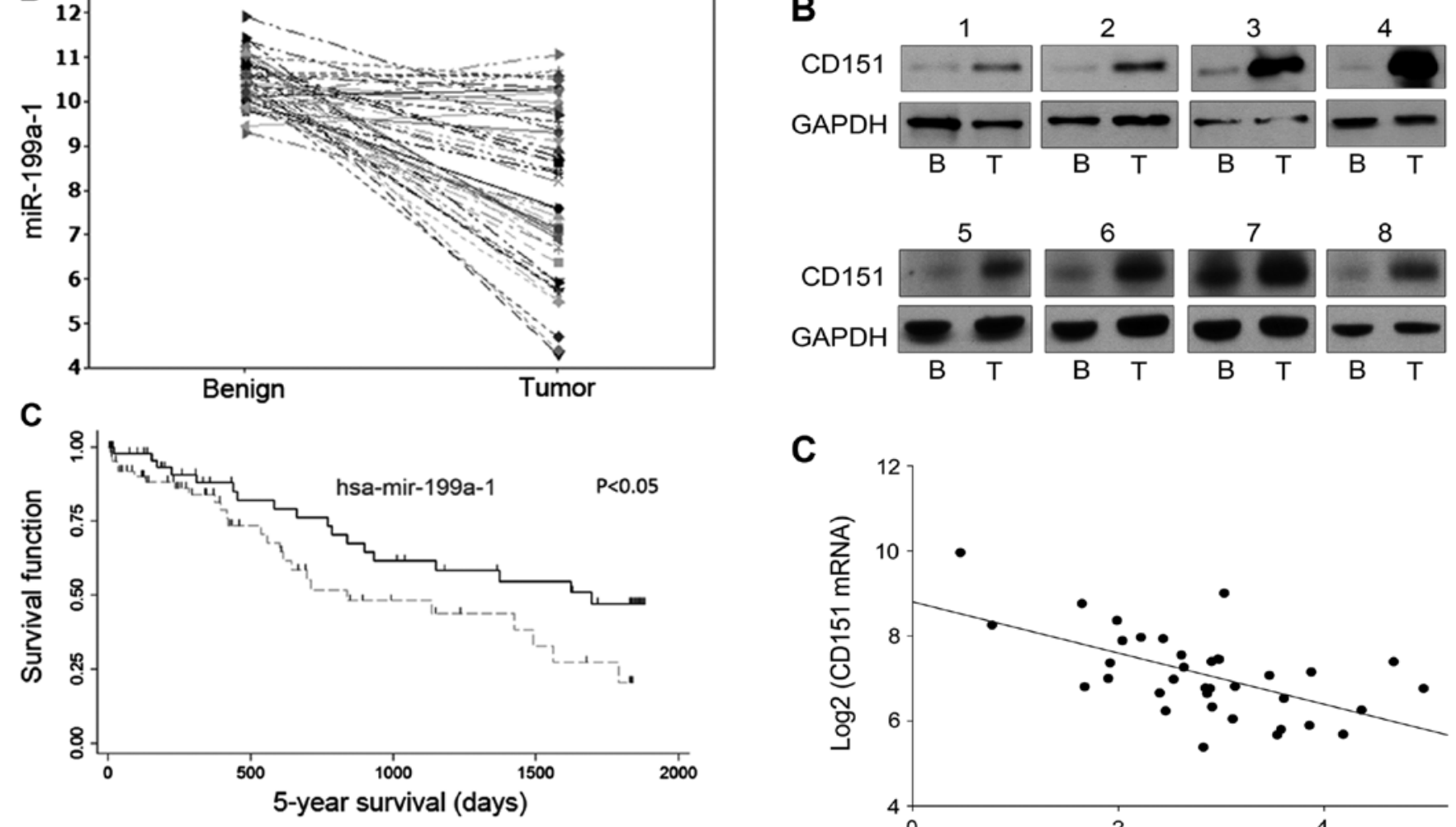

C

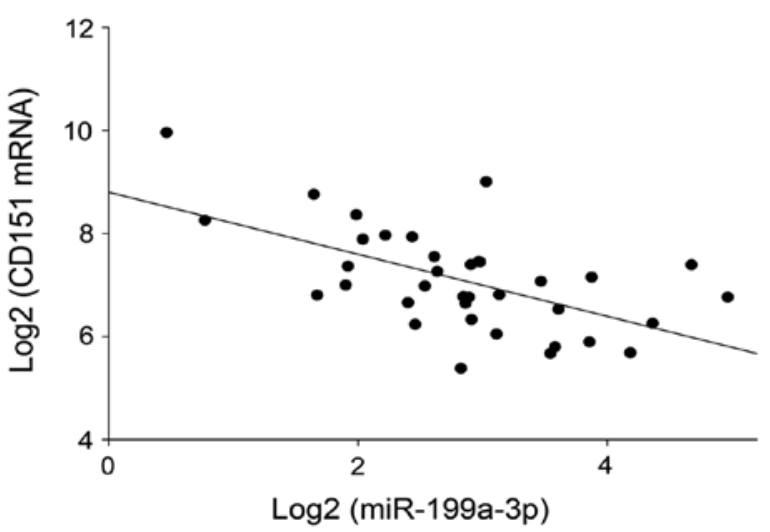

Figure 5. miR-199a-3p is reduced in HCC tissues and low miR-199a-1 expression correlates with poor survival. (A) miR-199a-3p expression relative to $18 \mathrm{~S}$ rRNA was measured by qRT-PCR in benign and tumor HCC specimens. (B) miR-199a-1 expression in 49 pairs of benign and HCC tissue from TCGA data set. (C) Correlation between miR-199a-1 expression and patient survival. Survival data from 141 patients from the TCGA liver hepatocellular carcinoma were analyzed by the log-rank test.

RNAs (Fig. 6C). While validating additional, putative target genes was beyond the scope of this study, it is of interest to note that miRBase predicts miR-199a-3p to regulate both integrin $\alpha 6$ (ITGA6) as well as integrin $\alpha 3$ (ITGA3). Thus, miR-199a-3p may play a key role in regulating two of the three factors involved in the CD151, integrin $\alpha 3 / 6$ and laminin 5 interaction, a complex that was reported to be critical for invasion and metastasis in HCC (14).

In addition to CD151, miR-199a-3p has been shown to regulate other tumor and metastasis promoting genes in $\mathrm{HCC}$ including c-met $(9,10)$, mTOR (9) and CD44 (11). The reduced

invasion and migration reported cannot be accounted solely by miR-199a-3p suppressing CD151 and the miRNA is likely suppressing known (i.e., c-met, mTOR and CD44) as well as unknown metastasis-related target genes. Since reduced miR-199a-3p expression in HCC would result in increased levels of these cancer promoting genes, it is conceivable that treating advanced HCC using miR-199a-3p oligo mimics could be used as a treatment for a disease that has very few treatment options. Treating HCC with miRNA mimics has great potential as it is well known that oligonucleotides accumulate in highly perfused organs such as the liver (26). In fact, 
miR-34 mimic is currently in phase I trial for the treatment of HCC (clinicaltrials.gov).

We previously showed that miR-199a-3p reduced the proliferation of CD44-positive HCC cell but not in CD44negative HCC cells by reducing CD44 protein levels (11). We report here that miR-199a-3p regulation of CD151 results in decreased invasion and migration, but CD151 does not directly regulate cell growth since siRNA knockdown of CD151 did not affect the proliferation of HCC cells. While CD44 is known to regulate cell adhesion and cell-cell interactions, it is also a well-known marker of stemness. In addition to its role in regulating metastasis-related mRNAs, miR-199a-3p also regulates HCV (27) and HBV replication (28). These findings, coupled with those reported herein, emphasize the critical role that a single deregulated miRNA may have on the cancer phenotype. Reduced miR-199a-3p could influence oncogenesis at various stages of development. Increased HCV and HBV replication may occur early on by reduced miR-199a-3p (28) followed by promoting proliferation in a CD44-dependent manner and increasing invasion and metastasis at later stages.

In conclusion, we showed that CD151 is involved in regulation of in vitro invasion and migration but not proliferation of HCC cell lines. miR-199a-3p, a miRNA that is significantly reduced in HCC, directly targets CD151. These data further implicate miR-199a-3p in the progression of HCC and suggests that oligonucleotide therapy using a miR-199a-3p mimic may be effective for treating advanced HCC.

\section{References}

1. Ferlay J, Soerjomataram I, Dikshit R, Eser S, Mathers C, Rebelo M, Parkin DM, Forman D and Bray F: Cancer incidence and mortality worldwide: Sources, methods and major patterns in GLOBOCAN 2012. Int J Cancer 136: E359-E386, 2015.

2. Raoul JL, Sangro B, Forner A, Mazzaferro V, Piscaglia F, Bolondi L and Lencioni R: Evolving strategies for the management of intermediate-stage hepatocellular carcinoma: Available evidence and expert opinion on the use of transarterial chemoembolization. Cancer Treat Rev 37: 212-220, 2011.

3. Aravalli RN, Steer CJ and Cressman EN: Molecular mechanisms of hepatocellular carcinoma. Hepatology 48: 2047-2063, 2008.

4. Calin GA and Croce CM: MicroRNA signatures in human cancers. Nat Rev Cancer 6: 857-866, 2006.

5. Gramantieri L, Ferracin M, Fornari F, Veronese A, Sabbioni S, Liu CG, Calin GA, Giovannini C, Ferrazzi E, Grazi GL, et al: Cyclin G1 is a target of miR-122a, a microRNA frequently downregulated in human hepatocellular carcinoma. Cancer Res 67: 6092-6099, 2007.

6. Hou J, Lin L, Zhou W, Wang Z, Ding G, Dong Q, Qin L, Wu X, Zheng Y, Yang Y, et al: Identification of miRNomes in human liver and hepatocellular carcinoma reveals miR-199a/b-3p as therapeutic target for hepatocellular carcinoma. Cancer Cell 19: 232-243, 2011.

7. Jiang J, Gusev Y, Aderca I, Mettler TA, Nagorney DM, Brackett DJ, Roberts LR and Schmittgen TD: Association of MicroRNA expression in hepatocellular carcinomas with hepatitis infection, cirrhosis, and patient survival. Clin Cancer Res 14: 419-427, 2008.

8. Murakami Y, Yasuda T, Saigo K, Urashima T, Toyoda H, Okanoue $\mathrm{T}$ and Shimotohno K: Comprehensive analysis of microRNA expression patterns in hepatocellular carcinoma and non-tumorous tissues. Oncogene 25: 2537-2545, 2006.

9. Fornari F, Milazzo M, Chieco P, Negrini M, Calin GA, Grazi GL, Pollutri D, Croce CM, Bolondi L and Gramantieri L: MiR-199a-3p regulates mTOR and c-Met to influence the doxorubicin sensitivity of human hepatocarcinoma cells. Cancer Res 70: 5184-5193, 2010
10. Kim S, Lee UJ, Kim MN, Lee EJ, Kim JY, Lee MY, Choung S, Kim YJ and Choi YC: MicroRNA miR-199a* regulates the MET proto-oncogene and the downstream extracellular signalregulated kinase 2 (ERK2). J Biol Chem 283: 18158-18166, 2008.

11. Henry JC, Park JK, Jiang J, Kim JH, Nagorney DM, Roberts LR, Banerjee S and Schmittgen TD: miR-199a-3p targets CD44 and reduces proliferation of CD44 positive hepatocellular carcinoma cell lines. Biochem Biophys Res Commun 403: 120-125, 2010.

12. Ke AW, Shi GM, Zhou J, Wu FZ, Ding ZB, Hu MY, Xu Y, Song ZJ, Wang ZJ, Wu JC, et al: Role of overexpression of CD151 and/or c-Met in predicting prognosis of hepatocellular carcinoma. Hepatology 49: 491-503, 2009.

13. Shi GM, Ke AW, Zhou J, Wang XY, Xu Y, Ding ZB, Devbhandari RP, Huang XY, Qiu SJ, Shi YH, et al: CD151 modulates expression of matrix metalloproteinase 9 and promotes neoangiogenesis and progression of hepatocellular carcinoma. Hepatology 52: 183-196, 2010.

14. Ke AW, Shi GM, Zhou J, Huang XY, Shi YH, Ding ZB, Wang XY, Devbhandari RP and Fan J: CD151 amplifies signaling by integrin alpha6beta1 to PI3K and induces the epithelial-mesenchymal transition in HCC cells. Gastroenterology 140: 1629-1641 e15, 2011.

15. Klosek SK, Nakashiro K, Hara S, Shintani S, Hasegawa H and Hamakawa H: CD151 forms a functional complex with c-Met in human salivary gland cancer cells. Biochem Biophys Res Commun 336: 408-416, 2005.

16. Arora H, Qureshi R and Park WY: miR-506 regulates epithelial mesenchymal transition in breast cancer cell lines. PLoS One 8: e64273, 2013

17. Han ZB, Yang Z, Chi Y, Zhang L, Wang Y, Ji Y, Wang J, Zhao H and Han ZC: MicroRNA-124 suppresses breast cancer cell growth and motility by targeting CD151. Cell Physiol Biochem 31: 823-832, 2013.

18. Wang X, Yu H, Lu X, Zhang P, Wang M and Hu Y: MiR-22 suppresses the proliferation and invasion of gastric cancer cells by inhibiting CD151. Biochem Biophys Res Commun 445: 175-179, 2014.

19. Tirmenstein MA, Nicholls-Grzemski FA, Schmittgen TD, Zakrajsek BA and Fariss MW: Characterization of nitric oxide production following isolation of rat hepatocytes. Toxicol Sci 53: 56-62, 2000.

20. Chen C, Ridzon DA, Broomer AJ, Zhou Z, Lee DH, Nguyen JT, Barbisin M, Xu NL, Mahuvakar VR, Andersen MR, et al: Real-time quantification of microRNAs by stem-loop RT-PCR. Nucleic Acids Res 33: e179, 2005.

21. Gebäck T, Schulz MM, Koumoutsakos P and Detmar M: TScratch: A novel and simple software tool for automated analysis of monolayer wound healing assays. Biotechniques 46: 265-274, 2009

22. Duan Q, Wang X, Gong W, Ni L, Chen C, He X, Chen F, Yang L, Wang P and Wang DW: ER stress negatively modulates the expression of the miR-199a/214 cluster to regulates tumor survival and progression in human hepatocellular cancer. PLoS One 7: e31518, 2012

23. Shi KQ, Lin Z, Chen XJ, Song M, Wang YQ, Cai YJ, Yang NB, Zheng MH, Dong JZ, Zhang L, et al: Hepatocellular carcinoma associated microRNA expression signature: Integrated bioinformatics analysis, experimental validation and clinical significance. Oncotarget 6: 25093-25108, 2015.

24. Sadej R, Grudowska A, Turczyk L, Kordek R and Romanska HM: CD151 in cancer progression and metastasis: A complex scenario. Lab Invest 94: 41-51, 2014.

25. Johnson JL, Winterwood N, DeMali KA and Stipp CS: Tetraspanin CD151 regulates RhoA activation and the dynamic stability of carcinoma cell-cell contacts. J Cell Sci 122: 2263-2273, 2009.

26. DeLong RK, Nolting A, Fisher M, Chen Q, Wickstrom E, Kligshteyn M, Demirdji S, Caruthers M and Juliano RL: Comparative pharmacokinetics, tissue distribution, and tumor accumulation of phosphorothioate, phosphorodithioate, and methylphosphonate oligonucleotides in nude mice. Antisense Nucleic Acid Drug Dev 7: 71-77, 1997.

27. Murakami Y, Aly HH, Tajima A, Inoue I and Shimotohno K: Regulation of the hepatitis $\mathrm{C}$ virus genome replication by miR-199a. J Hepatol 50: 453-460, 2009.

28. Zhang GL, Li YX, Zheng SQ, Liu M, Li X and Tang H: Suppression of hepatitis B virus replication by microRNA199a-3p and microRNA-210. Antiviral Res 88: 169-175, 2010. 